MATEC Web of Conferences 11,02008 (2014)

DOI: $10.1051 /$ matecconf / 20141102008

(C) Owned by the authors, published by EDP Sciences, 2014

\title{
Conception et construction d'un ouvrage de soutènement (en blocs de béton) suspendus et soutenus par des contres poids
}

\author{
Tahar Ayadat \\ Dept. of Civil Engineering, Prince Mohammad Bin Fahd University, Al-Khobar (Saudi Arabia)
}

\begin{abstract}
You should leave $10 \mathrm{~mm}$ of space above the abstract and $15 \mathrm{~mm}$ after the abstract. The heading Abstract should be typed in bold 9-point Arial. The body of the abstract should be typed in normal 9-point Times in a single paragraph, immediately following the heading. The text should be set to 1.15 line spacing. The abstract should be centred across the page, indented $15 \mathrm{~mm}$ from the left and right page margins and justified. It should not normally exceed 300 words.
\end{abstract}

\section{Introduction}

Une étude géotechnique concernant la conception, l'analyse de stabilité et la construction des murs de soutènement en blocs projetés dans l'entourage du ponceau a été effectuée. La présente étude rentre dans le cadre du projet de remplacement du ponceau P-7796 du ruisseau Doncaster, sur la rue De l'Église, dans la municipalité de Val-David, au Québec.

Les objectifs de l'étude géotechnique étaient de déterminer la méthode de conception et de construction des murs de soutènement en blocs (murs poids) en tenant compte de la spécificité des lieux, tel que le manquement d'espace (endroits d'emplacement très restreints).

Le présent article renferme toutes informations utiles obtenues de l'étude géotechnique réalisée, notamment les conditions de sol et d'eau souterraine prévalant au site du ponceau, ainsi que la méthode proposée pour la conception des murs, nos analyses de stabilités, et nos recommandations concernant les étapes de constructions des dits murs.

\section{Description et emplacement des murs de soutennement}

Tel que mentionné précédemment, les murs de soutènements sont projetés dans l'entourage du ponceau P-7796. Ces murs sont des étançonnements temporaires rentrant dans le cadre du projet de remplacement du ponceau du ruisseau Doncaster, sur la rue De l'Église, dans la municipalité de Val-David, au Québec.
Il faut noter que ces étançonnements temporaires ont été conçus en murs poids (murs en blocs), vu que l'installation d'autres types de murs tels que les murs Berlinois ou les palplanches provoquent des bruits dépassant les 75 décibels fixés par les autorités compétentes. En outre, l'adoucissement de la pente du terrain, caractérisée par un angle droit, est pratiquement inconcevable, vu que le terrain adjacent est une propriété privée.

Ces murs de soutènement sont situé dans le coté sud-est de la rue De l'Église ou du ponceau projeté. Ils sont localisés des deux cotés du ruisseau Doncaster (un mur de chaque coté), parallèlement aux murs du ponceau. L'extrémité sud du mur (A) est placée à $\pm 0,60 \mathrm{~m}$ du point 13 , alors que le mur (B) est à plus d'un mètre du point 20. L'emplacement de ces deux murs est bien indiqué sur la figure 1.

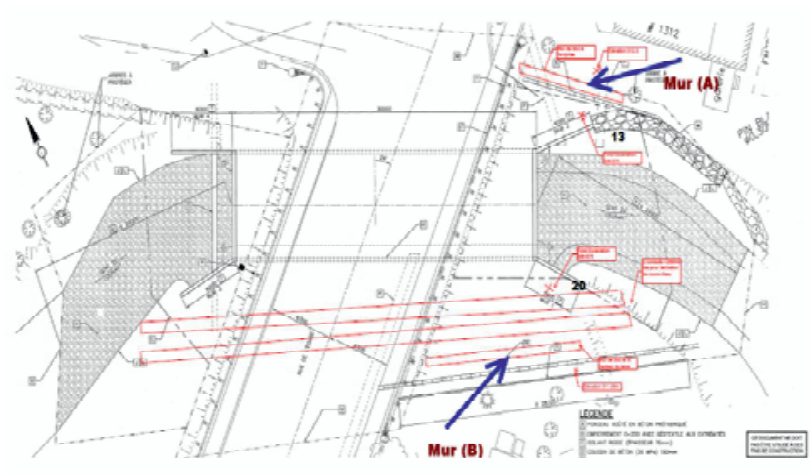

Fig. 1. Emplacement des murs en blocs peojetés. 
Les dimensions approximatives des murs sont 8,5 $\mathrm{m}$ de longueur et $4,5 \mathrm{~m}$ de hauteur pour le mur (A), et d'environ 1,0 à $2,0 \mathrm{~m}$ de hauteur et 8,0 à $10,0 \mathrm{~m}$ de longueur pour le mur (B). Ils sont construits par des blocs de béton de $0.61 \mathrm{~m}$ de hauteur, $0,915 \mathrm{~m}$ de largueur et $1,22 \mathrm{~m}$ de longueur.

\section{Données géotechniques utilisés pour la conception}

Les travaux de reconnaissance sur le terrain ont été effectués du 17 au 20 avril 2006. Ils ont consisté en l'exécution de 2 forages conventionnels avec échantillonnage numérotés F-1 et F-2.

Une description générale des différents horizons de sol rencontrés sur le site est présentée comme suit :

1- Sol organique: à partir de la surface, les forages ont traversé $100 \mathrm{~mm}$ de sol organique

2- Remblai: sous le sol organique, les 2 forages ont traversé un remblai granulaire. $\mathrm{Ce}$ remblai possède des épaisseurs respectives de 4,5 m (F-1) et de 3,4 m (F-2). Il consiste en un sable graveleux avec un peu de silt (SM). Les valeurs de l'indice de pénétration $\left(N_{s p t}\right)$ obtenues varient entre 6 et 19 , ce qui correspond à une compacité lâche à moyenne.

3- Dépôt de sable: immédiatement sous le remblai, les deux forages ont traversé un dépôt de sable contenant des débris de bois correspondant probablement au lit du ruisseau. Cet horizon présente des épaisseurs de $5,40 \mathrm{~m}$ (F-1) et $7,17 \mathrm{~m}$ (F-2). La composition de ce dépôt naturel est similaire à celle des matériaux du remblai sus-jacent. En général, les valeurs de l'indice de pénétration obtenues varient entre 9 et 36 , ce qui correspond à une compacité lâche à dense.

4- Roc: il a été atteint et carotté dans les deux forages F-1 et F-2 à partir des profondeurs respectives de $10,00 \mathrm{~m}$ et $10,67 \mathrm{~m}$. Il consiste en une monzodiorite à grain moyen à gossier.

Le niveau de l'eau souterraine, mesuré le 20 avril 2006 dans des tubes d'observation installés dans les deux forages, est situé à la profondeur 3,60 m (F-1) et 2,60 m (F-2).

Le nouveau ponceau est constitué d'une structure en béton de type CON/SPAN dont le radier serait implanté à environ $5 \mathrm{~m}$ de profondeur sous le niveau de la chaussée.
La capacité portante aux états limites d'utilisation préconisée pour la conception des fondations placées dans le dépôt naturel de sable est présentée dans le tableau 1.

Tableau 1. Capacité portante aux états limites d'utilisation

\begin{tabular}{|c|c|c|c|c|c|c|c|}
\hline & \multicolumn{6}{|c|}{ Largeur de l'empâtement (m) } \\
\hline $\begin{array}{c}\text { Capacité } \\
\text { portante } \\
\text { l'ELUT } \\
\text { (kPa) }\end{array}$ & 0,0 & 0,5 & 1,0 & 1,5 & 2,0 & 2,5 & 3,0 \\
\cline { 2 - 8 } & 400 & 400 & 370 & 330 & 280 & 270 & 250 \\
\hline
\end{tabular}

Le remblayage derrière les murs du ponceau peut être effectué avec les matériaux d'excavation ou à l'aide d'un matériau MG 112 (NQ 2560-114/2002). La pression horizontale sur les murs due au poids a été calculée en adoptant pour le remblai un poids volumique $\gamma=18 \mathrm{kN} / \mathrm{m}^{3}$ et un angle de frottement interne $\varphi=30^{\circ}$. Le coefficient de poussée active a été déterminé en tenant compte de la pente du remblai placé à l'arrière des murs.

Les sols de fondations situés sous la nappe phréatique sont des sols granulaires lâche et de compacité moyenne à dense, donc probablement liquéfiables. Cependant, étant donné que les structures étudiées (murs de soutènement en blocs) sont temporaires aucune vérification de susceptibilité à la liquéfaction n'a été effectuée.

\section{Analyse de stabilité}

La particularité de ce projet se résume dans le fait que :

- Les endroits d'emplacement des ouvrages sont très restreints (par exemple, la largeur de l'espace réservé à l'installation du mur A est de l'ordre de $0,60 \mathrm{~m}$ ),

- L'utilisation de murs Berlinois ou de palplanches n'est pas envisageable à cause du bruit (limité à 75 décibels dans les lieux),

- L'adoucissement des pentes est inconcevable (le terrain adjacent appartient à un privé),

- Le budget alloué à la solution adoptée est limité.

Donc, on était contraint de procéder par l'utilisation de murs en blocs (mur poids). Cependant, vu la contrainte d'espace concernant l'endroit d'emplacement, les murs en blocs doivent être minces. Ceci nous oblige à prévoir des techniques 
supplémentaires pour assurer la stabilité des dits murs, notamment du mur (A). En conséquence, les trois profils (ou sections) suivants ont été proposés et analysés (figure 2).

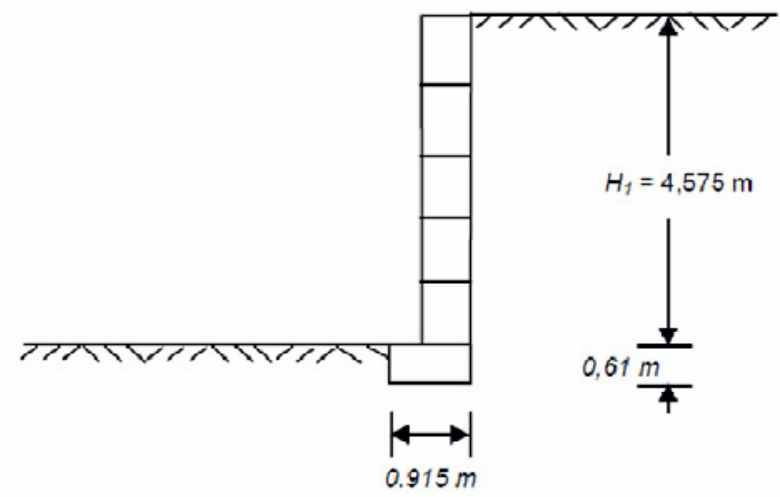

Fig. 2a. Profil P-1 du mur en bloc (A).

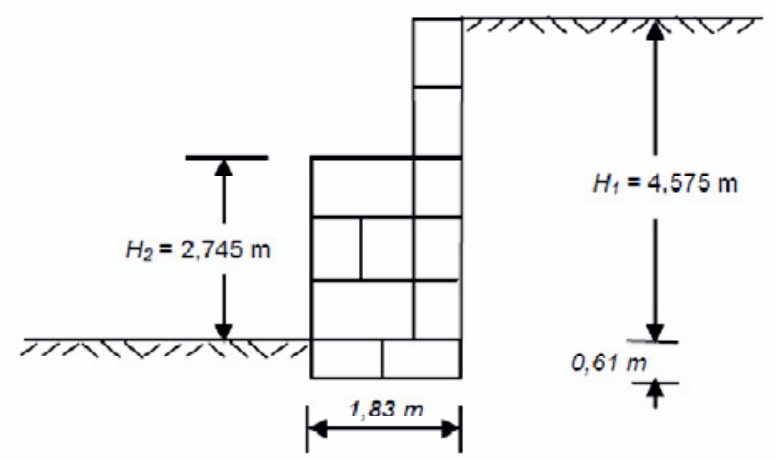

Fig. 2b. Profil P-2 du mur en bloc (A).

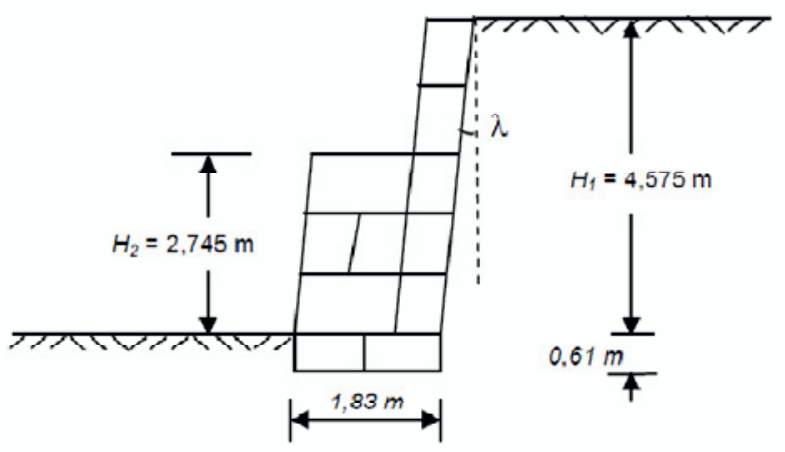

Fig. 2c. Profil P-3 du mur en bloc (A)

Généralement, l'analyse de stabilité d'un mur de soutènement comprend la stabilité vis-à-vis le glissement, le renversement et le poinçonnement (ou capacité portante du sol de fondation). Les calculs de vérification ou de stabilité effectués pour les trois profils présentés dans la figure 2 sont résumés dans le Tableau 2.
Tableau 2. Coefficients de sécurité de l'analyse de stabilité.

\begin{tabular}{|c|c|c|c|c|}
\hline Profile & Figure & $\begin{array}{c}\text { Stabilité vis- } \\
\text { a-vis le } \\
\text { glissement }\end{array}$ & $\begin{array}{c}\text { Stabilité } \\
\text { vis-à-vis le } \\
\text { renverseme } \\
\text { nt }\end{array}$ & $\begin{array}{c}\text { Stabilité } \\
\text { vis-à-vis le } \\
\text { poinçonne } \\
\text { ment }\end{array}$ \\
\hline P-1 & $2 a$ & 0,58 & 0,6 & $\mathrm{e}>\mathrm{L} / 6$ \\
\hline P-2 & $2 b$ & 1,2 & 1,85 & 1,94 \\
\hline P-3 & $2 c$ & 1,69 & 2,27 & 2,88 \\
\hline \multicolumn{2}{|c|}{$\begin{array}{c}\text { Valeurs } \\
\text { admissibles } \\
\text { (FHWA, 1990) }\end{array}$} & 1,5 & 2,0 & 2,0 \\
\hline
\end{tabular}

D'après les calculs des coefficients de sécurité des trois sections, résumées dans le Tableau 2, le profil P3 a été retenu à condition de prévoir impérativement les précautions suivantes :

- Les blocs de la partie principale du mur (partie en contact avec le terrain à soutenir) doivent être collés ensemble, par exemple par du mortier ou des ancrages mécaniques, pour agir comme un seul élément. En outre, cette partie doit être colée à la série de blocs ancrés dans le sol (série de base).

- Cette partie du mur doit être attachée à d'autres blocs placés en surface; à travers des câbles métalliques tel que indiqué sur la figure 3. Trois points d'attachement sont envisageable (un au centre et un à chacune des extrémités du mur) ou les câbles doivent traversés des trous percés transversalement dans les blocs, tel que indiqué dans les coupes $\mathrm{AA}, \mathrm{BB}$ et $\mathrm{CC}$ de la figure 3 , ou bien de faire passer les câbles à travers les crochets des blocs.

- Un angle d'inclinaison du mur avec la verticale $\lambda=6^{\circ}$ a été considéré durant les calculs. Cependant, un angle $\lambda \geq 10^{\circ}$ est préconisé pour augmenter la stabilité.

Finalement, en se basant sur le fait que la stabilité du système augmente d'avantage si les blocs sont placés ou posés sur leur côté le plus large, la disposition finale retenu du mur (A) pour le présent projet est représentée sur la figure 4. Dans ce cas, les câbles sont fixés à travers les crochets de blocs fait de barres d'armatures pliés ou de câbles, tel que indiqué sur la figure 4 (le câble doit être tendu et fixé à tous les crochets). En outre, un remblai granulaire est placé entre le mur de blocs et le terrain. 


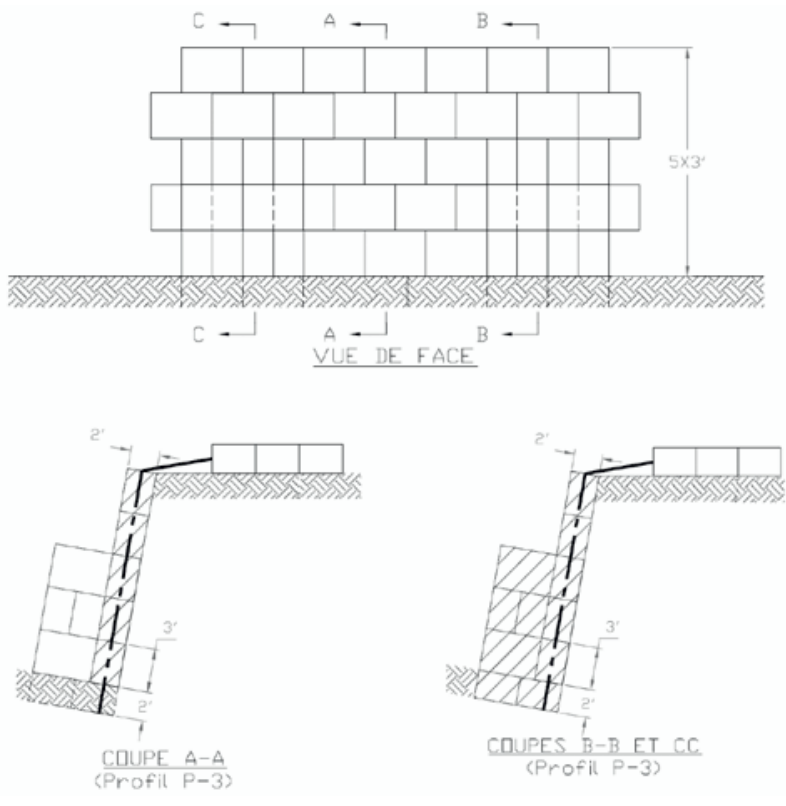

Fig. 3. Vue de face et les differentes coupes du mur en blocs

(A)

Concernant le mur (B), il est a noté que vu la disponibilité de l'espace à l'endroit d'emplacement et vu que le sol en place profilé en forme de talus est stable, il n'y avait pas recours à un mur d'une grande hauteur. Dans ce cas, un ou deux séries de blocs non inclinées ont été acceptés comme solution.

\section{Etape de construction du mur en bloc}

\section{(A)}

La disposition retenue du mur (A) est représentée sur la figure suivante (figure 4). Les étapes de construction du dit mur sont représentées sur la figure 5 et résumer comme suit :

- Étape 1: Emplacement des blocs de base (ancrés dans le sol de fondation)

- Étape 2 : Construction de la $1^{\text {ere }}$ série du mur principal

- Étape 3 : Construction de la $1^{\text {ere }}$ série du mur contre poids (mur d'appui)

- Étape 4: Construction de la $2^{\text {eme }}$ série du mur principal

- Étape 5: Construction de la $2^{\text {eme }}$ série du mur contre poids

- Étape 6: Construction de la $3^{\text {eme }}$ série du mur principal

- Étape 7: Construction de la $3^{\text {eme }}$ série du mur poids;

- Étape 8: Construction de la $4^{\text {eme }}$ série du mur principal;

- Étape 9: Construction de la $4^{\text {eme }}$ série du mur poids;

- Étape 10 à 12: Construction de la $5^{\mathrm{eme}}, 6^{\mathrm{eme}}$ et $7^{\text {eme }}$ série du mur principal.
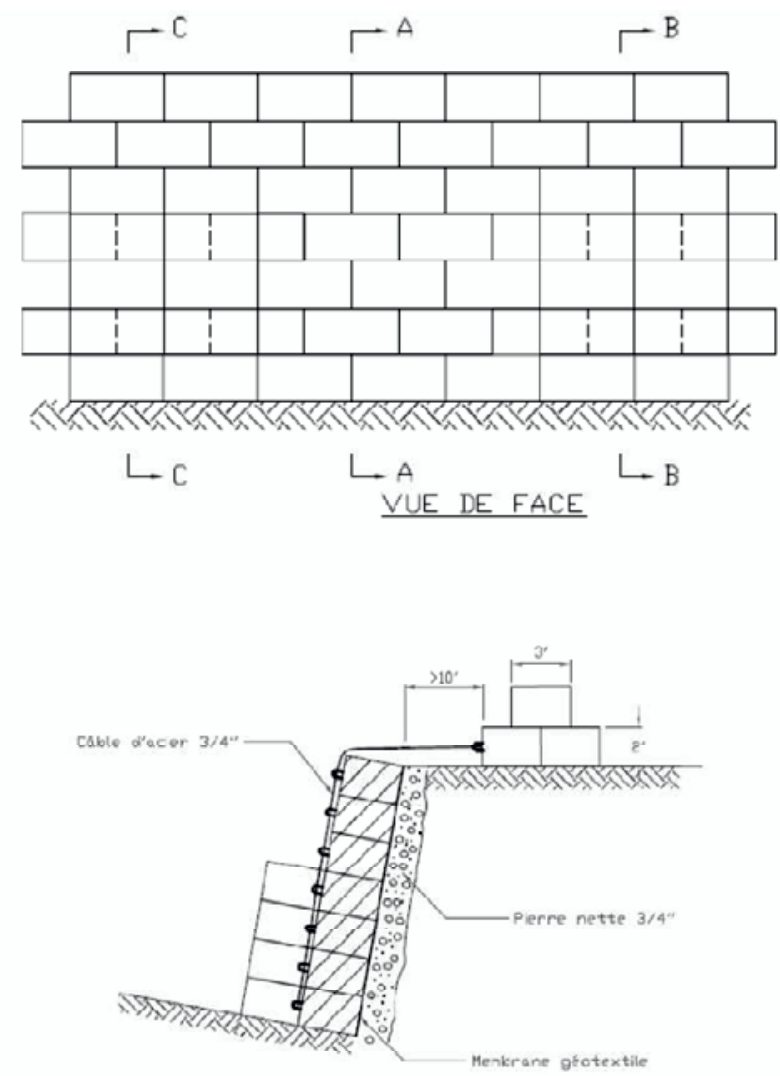

$\frac{\text { CDUPE } A-A}{\text { (PRDFIL P-3) }}$

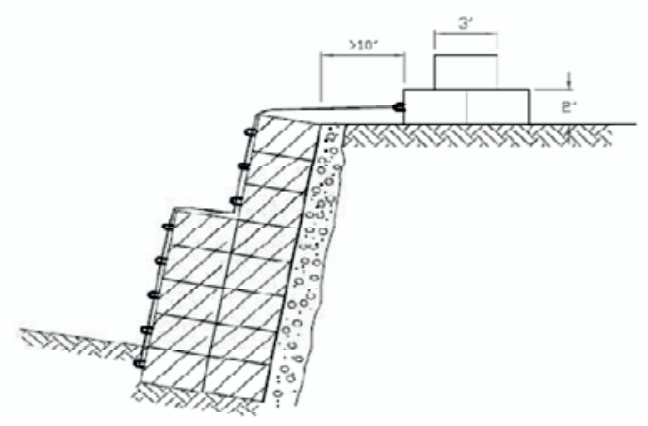

CIUPF $\mathrm{B}-\mathrm{B}$ FT $\mathrm{C}-\mathrm{C}$

Fig. 4. Vue de face et les différentes coupes du profil retenu du mur en blocs (A)

\section{Références}

1. Etude géotechnique: Construction de murs de soutènement (en blocs) projetés dans l'entourage du ponceau TTOG de la rue l'Eglise à Val-David, F102517-001, octobre 2002.

2. Etude géotechnique: Construction du ponceau TTOG de la rue 1'Eglise à Val-David, G06162-B-rap-001, avril 2006. 


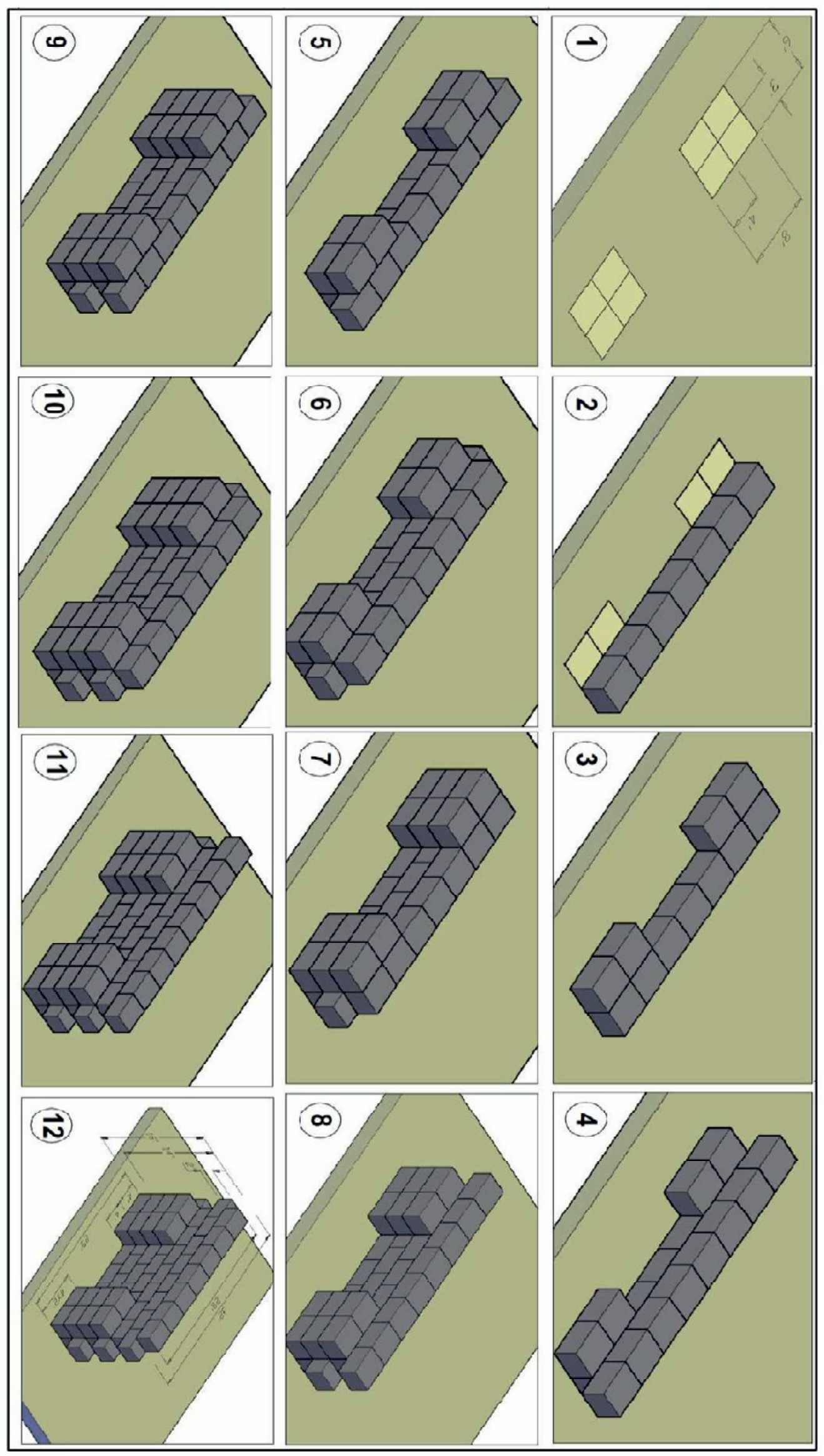

Fig. 5. Les étapes de construction du mur en blocs (A) 